\title{
Post-operative Complications Following Emergency Laparotomy are Common and Associated with Increased Late Mortality - a Retrospective Multi- centre Study.
}

shivam sharma

University of Birmingham https://orcid.org/0000-0002-1741-2111

Joseph Alderman

University of Birmingham

Dhruv Parekh

University of Birmingham

David Thickett

University of Birmingham

Jaimin Patel ( $\sim$ jaimin.patel@uhb.nhs.uk)

University of Birmingham https://orcid.org/0000-0003-4120-3923

\section{Research Article}

Keywords: Post-operative Pulmonary Complications, emergency laparotomy, mortality, re-admissions, outcomes.

Posted Date: June 1st, 2021

DOI: https://doi.org/10.21203/rs.3.rs-428635/v1

License: (c) (i) This work is licensed under a Creative Commons Attribution 4.0 International License. Read Full License

Version of Record: A version of this preprint was published at Journal of Surgery and Research on January 1st, 2022. See the published version at https://doi.org/10.26502/jsr.10020198. 


\section{Abstract}

\section{Background}

The first National Emergency Laparotomy Audit (NELA) highlighted that morbidity and mortality from emergency surgery remains elevated especially in high-risk patients defined as a P-POSSUM mortality $\geq$ $5 \%$ and ASA $\geq 3$. The incidence of postoperative pulmonary complications (PPCs) are thought to be high following emergency laparotomy but no recent studies have evaluated the incidence or consequences of PPC following emergency laparotomy in the UK.

\section{Methods}

A retrospective cohort study was conducted at University Hospital Birmingham and Heartlands Hospital, Birmingham, to investigate the incidence of PPCs following emergency laparotomy. The NELA databases from the two Trusts were used to identify patients. Patients were retrospectively screened for the development of PPCs using the validated Melbourne Group Scale. Data was analysed using Chi-squared test for categorical data and continuous data displayed as medians with statistical analysis from a Mann-Whitney U test.

Results

A total of 362 correctly coded patients were identified. High-risk patients accounted for $62 \%(226)$ of the cohort. These patients were older $(p<0.001)$ and had higher baseline lactate $(p=0.04)$ and creatinine levels $(p=0.003)$. Median P-POSSUM mortality was $10.6 \%(5.6-31.4 \%)$ with $76.4 \%$ of patients having an $A S A \geq 3$. These patients had an increased length of stay $(p<0.001)$ and accounted for nearly all the deaths (42 vs. $2 ; p<0.001$ ). The incidence of PPCs was $37 \%$, again the incidence was greater in the highrisk group ( $37 \%$ vs. $6 \%$ p < 0.001). Development of a PPC was associated with an increased length of stay (17 d vs. $9 \mathrm{~d} ; \mathrm{p}<0.001)$ as well as a 90,180 and 360 day mortality.

\section{Discussion}

This study demonstrates that the sub-group of patients deemed 'high-risk' are at greatest risk of developing a PPC and consequently have an increased length of stay and an increased 90, 180 and 360 day mortality. This allowed us to identify a group of patients at high risk of PPC who we can target with potential novel therapies such as high-flow nasal cannulae oxygen in clinical trials to reduce mortality and morbidity.

\section{Background}

Emergency abdominal surgery, often termed emergency laparotomy, is a common surgical procedure undertaken in the United Kingdom (U.K), with approximately 30000 procedures annually(1). Patients presenting for emergency abdominal surgery are heterogeneous and present with diverse pathology, resulting in challenges for the surgical, anaesthetic and critical care team that manage them. Emergency 
laparotomy, by its very nature is high-risk surgery, with an estimated 30 -day mortality in the U.K of $11 \%$, which is over 10 times greater than the mortality of patients undergoing major elective surgery (e.g. cardiac, vascular and oncological surgery) and a median hospital length of stay (LOS) of 12-days(2,3).

Risk factors associated with poorer outcomes from emergency laparotomy have been identified by the National Emergency Laparotomy Audit (NELA) and include advancing age, with each decade above the age of 50 being associated with increasing risk. Additional risk factors include an American Society of Anaesthesia (ASA) status of 3 or more and Portsmouth- Physiological and Operative Severity Score for the enumeration of Mortality and morbidity (P-POSSUM) risk of death of greater than $5 \%(3)$.

The development of post-operative pulmonary complications (PPCs) is a composite definition for a variety of respiratory complications that occur following surgery. They range from clinically significant bronchospasm and atelectasis, through to the development of pneumonia and the acute respiratory distress syndrome (ARDS)(4). The incidence following elective major abdominal surgery has been estimated at $11.9 \%$ and is associated poorer outcomes with increased length of hospital stay, increased re-admissions and a higher mortality(5). Although emergency surgery is well established as a significant risk factor for the development of PPCs, the incidence is not well established.

This study aimed to establish the incidence of PPCs in a cohort of patients undergoing emergency laparotomy and the consequences on patient outcomes.

\section{Methods}

\section{Design and Setting}

A retrospective analysis was conducted of patient that underwent emergency laparotomy between December 1st 2014 and November 31st 2015 at University Hospital Birmingham National Health Service (NHS) Foundation trust and Heartlands Hospital, Birmingham (part of the Heart of the England NHS Foundation Trust). The two hospitals combined serve a population of approximately 1 million people, have a total of 3000 hospital beds, with 120 critical care beds and offer acute surgical services. The study did not require ethical approval or research registration. This was confirmed by the online National Research Ethics Survey decision tool and the research and development departments at the University of Birmingham(6).

Patients were identified using the NELA databases for each NHS trust which collects local hospital data for emergency laparotomy, however this is not a fully comprehensive database. Therefore, in addition to this, each hospital's electronic patient records (EPR) were searched over the same timeframe to crossreference patients and collect patients that had not been initially identified using the NELA database.

\section{Data Collected}


Individual patient data was collected using the NELA database which collects information to include baseline demographics, date of operation, risk stratification using ASA score, P-POSSUM, serum lactate and serum creatinine, operative details, critical care service utilisation post-operatively and in-hospital outcomes. This was supported by electronic EPR systems at each trust to collect data for PPCs, unplanned hospital re-admissions and mortality at 90-days, 180-days and 365-days.

\section{Definition Of High-risk And Low-risk Patients}

As described previously patients presenting for emergency surgery are heterogeneous with different groups of patients having variable outcomes. Patients were therefore categorized as high or low-risk based upon either an an ASA $\geq 3$ or a P-POSSUM predicted mortality of $\geq 5 \%$. This categorization was based on evidence from 30000 cases analysed by the NELA group, that suggested that adverse outcomes occur if a patient meets any one of these three variables(3).

\section{Diagnosis Of Ppcs}

PPCs were identified using the Melbourne Group Score. This is a validated daily screening tool for identifying patients with PPCs and has been used previously in patients undergoing major abdominal and thoracic surgery(7). The score consists of 8 dichotomous factors that include microbiological, clinical and physiological parameters, with a score of 4 or more indicating the development of a clinically significant PPCs that are likely to adversely affect the patient's clinical course (Fig. 1). Patients EPRs were screened daily from the day of operation till the seventh post-operative day for PPCs using the Melbourne Group Score. The daily score was recorded and where a patient met the diagnosis of a PPC the postoperative day of occurrence was recorded.

\section{Statistical Analysis}

Continuous data was analysed for normality using a D'Agostino and Pearson omnibus normality test. All data were non-parametric and therefore all continuous is represented as median and inter-quartile ranges $(\mathrm{IQR})$, with comparative tests between groups analysed using a Mann-Whitney $U$ test. Categorical data are represented as numbers and proportions with any comparative tests performed using a Fisher's exact or Chi-squared test. Kaplan-Meier analysis and odd's ratio were performed to quantify the consequences of PPC development on survival and re-admissions. These were calculated using a log-rank Mantel-Cox Chi-squared test. All data analysis was performed using GraphPad PRISM version 6 (La Jolla, USA). Additionally, to identify factors that may predict the development of PPCs Cox-logistical regression was carried out using SPSS. Significance was taken as a p-value of less than 0.05 .

\section{Results}


A total of 437 patients were identified as having undergone emergency laparotomy between the 1st of December 2014 and 31st November 2015. 75 cases were excluded due to duplication or incorrect coding of the procedures. Of the 362 remaining patients, 136 were classified as low-risk patients and 226 as being high-risk (Fig. 2).

\section{Demographics}

The median age of the entire cohort was 62 years (IQR 45-74 years), with $47 \%(n=170)$ of patients being male. The majority of patients $(64 \% \mathrm{n}=230)$ had an ASA $\geq 3$ with a median P-POSSUM score of $6 \%(2.3-$ $19.3 \%) .48 \%(n=174)$ of patients were admitted directly to critical care units (CCU) following surgery. The median length of hospital stay was 13 days (IQR 6-25), with a 30-day mortality of $12 \%(n=44)$. (see Table 1)

High-risk patients were older (67 years $\{$ IQR $51-77\}$ vs. $48\{$ IQR 38-66\}; $p<0.001$ ) and had higher PPOSSUM predicted mortality scores (10.6\% \{IQR 5.6-31.3\} vs. $2 \%\{$ IQR $1.3-3.1\} ; p<0.001)$. These patients also had higher plasma creatinine $(74 \mathrm{mmol} / \mathrm{L}\{59-104 \mathrm{mmol} / \mathrm{L}\}$ vs. $70 \mathrm{mmol} / \mathrm{L}\{58-80 \mathrm{mmol} / \mathrm{L}\} ; \mathrm{p}$ $=0.003)$ and lactate levels $(1.8 \mathrm{mmol} / \mathrm{L}\{1.2-3.5 \mathrm{mmol} / \mathrm{L}\} \mathrm{vs} .1 .5 \mathrm{mmol} / \mathrm{L}\{1.1-2.6 \mathrm{mmol} / \mathrm{L}\} ; \mathrm{p}=0.044)$ at the time of surgery. High-risk patients had significantly poorer outcomes with an increased length of hospital stay (17days $\{$ IQR $9-32\}$ vs. 9days $\{$ IQR $6-15\} ; p<0.001)$ and an increased 30 -day mortality $(14 \%$ vs. $1.4 \% ; p<0.001)$.

\section{Incidence Of Ppcs}

The incidence of PPCs within the entire cohort was 30\% $(n=108)$. The burden of PPCs were almost entirely in the high-risk patients with an incidence of $37 \%(n=84)$, with only $6 \%(n=8)$ of the low-risk patients developing a PPC. The demographics of patients who were diagnosed with and without a PPC are shown in Table 2. There were no significant differences between these patients, although patients who did develop a PPCs showed a trend towards an increased pre-operative P-POSSUM predicted mortality scores and were more likely to be admitted directly to CCU ( $85 \%$ vs. $71 \%$; $p=0.02)$. Importantly, there were no differences between these two cohorts in baseline respiratory or cardiovascular disease as measured by the P-POSSUM risk score. The commonest post-operative day to develop a PPC was day 3(median; IQR 1-4days).

\section{Outcomes In Patients With Ppcs}

Patients that developed a PPC during the first 7 post-operative days had poorer outcomes compared to patients who did not develop a PPCs. This included a median increase in CCU length of stay by 1-day ( $\mathrm{p}$ $0.002)$ and a median increase in hospital length of stay of 3days $(p=0.01)$. There was no difference in 30-day mortality, however there was a significant increase in both 90, 180 and 365-day mortality (see 
Fig. 3). In addition, the number of re-admissions in patients who developed a PPC was increased at similar time-points.

\section{Multivariate Analysis}

Cox's multiple regression was performed on factors that may have predicted the development of a PPC in the high-risk cohort. No individual factors predicted the likelihood of PPC development in the high-risk cohort.

\section{Discussion}

Emergency abdominal surgery by its very nature is associated with increased morbidity and mortality. This study demonstrates that the well validated ASA and P-POSSUM scoring tools in conjunction with age can be used to identify patients at high risk of developing PPCs. Importantly we demonstrate that the patients who develop a PPC overall have poorer outcomes and consume more resources. Although, inhospital or 30-day mortality was no different, patients who developed a PPC did stay in CCU for longer and have prolonged hospital stays compared to those who did not develop a PPC. Longer term outcomes, were also poorer, with unplanned hospital readmissions and mortality increased at all time points.

The pathophysiology of PPCs following emergency surgery is multi-factorial and complex. Post-operative pain following surgery is implicated as this prevents patients from ventilating dependent portions of the lung(8). The site of the surgical incision is also relevant with incisions that extend above the umbilicus, or sub-phrenic incisions causing significant respiratory compromise. Control of pain is a key principal of anaesthesia and post-operative management and regional anaesthesia, especially the use of neuro-axial blockade, can effectively mitigate against these factors in the elective operative setting. In emergency surgery, neuro-axial blockade may be relatively contra-indicated due to physiological and coagulation abnormalities and as such are utilised less frequently.

Patients undergoing emergency abdominal surgery are heterogenous with multiple pathologies leading to their definitive operative procedures. These can range from blunt or penetrating trauma causing internal visceral damage with/without major haemorrhage, to contained bowel obstruction to fulminant peritonitis or ischaemic bowel. An added complexity is the underlying disease processes in non-trauma patients are varied, such as bowel cancer, inflammation (diverticulitis) and anatomical abnormalities (hernias/volvulus). Furthermore, these patients may present with poorly controlled co-morbid disease, such as diabetes, ischaemic heart disease, lung diseases, which cannot be significantly pre-optimized prior to surgery, increasing the chances of peri- and post-operative complications.

Studies in cohorts of elective patients undergoing major elective surgery have demonstrated similar finding to our current study $(9,10)$. These studies suggest that elective patients who develop PPCs have increased length of stay and an increased 30 -day mortality $(11,12)$. Our study supports these findings; however it demonstrates that its incidence is much higher and that outcomes in the longer term (through 
to 1-year) are much worse. Interestingly our study showed that PPCs were one of the major sources of morbidity, with surgical site infections and acute myocardial ischaemia being infrequently reported.

PPCs are a composite definition, with the validated Melbourne Group Score designed to select the clinically significant infection and atelectasis, which arguably are the most serious and often lead to acute lung injury (ALI) and ARDS. The development of nosocomial pneumonia and infections has been shown in several studies to increase length of stay in CCU and also in-hospital, however the precise reasons why longer-term outcomes are poorer is uncertain. It has been suggested that episodes of infection/sepsis cause prolonged immune dysfunction placing survivors of infection/sepsis at higher risk of subsequent infections following discharge from hospital(13). This may explain the increased rates of emergency re-admissions and later mortality in these patients.

PPCs following emergency abdominal surgery have been poorly evaluated, with many studies and trials concentrating on elective surgical populations, where significant risk-factors for PPCs (e.g. smoking and respiratory disease) can be potentially $\operatorname{modified}(9,10)$. The high incidence of PPCs in the high-risk cohort suggests that potential therapies should be targeted at them to attempt to modify their risks of pulmonary complications and that by doing so may improve their longer-term outcomes. Peri-operative ventilation strategies to modify PPCs have been investigated. The landmark IMPROVE trial investigated the use of lung protective ventilation using positive end-expiratory pressure (PEEP) and low tidal volumes and showed significantly reduced incidence of both pulmonary and non-pulmonary infection(14). Whilst the PROVILHO study investigated the use of high versus low PEEP and demonstrated no difference, but importantly did suggest that high PEEP strategies were associated with increased cardiovascular compromise(15). Both these studies have potentially important implications in emergency abdominal surgery, however they only recruited patients undergoing major abdominal surgery. The use of protective ventilation in emergency surgery would seem intuitive and supported by evidence in ventilated critically ill patients without ALI/ARDS. Indeed, a prospective observational study of ventilation practices did demonstrate that anaesthetists adopted a more protective strategy in this cohort of patients(16).

Post-operative interventions have also been evaluated, with the recently completely OPERA trial, investigated the use of high-flow nasal oxygen in patients undergoing major abdominal surgery, but found no difference in oxygenation 4 hours post-operatively (primary outcome), nor in any of their clinical secondary outcomes, which included PPCs and LOS(17). This study, however recruited very few emergency surgical patients.

The retrospective nature of this study clearly has clear limits, with perhaps the incidence of PPCs begin over-estimated and with the inability to account for confounding factors. Additionally, due to the retrospective review of EPR and collected data some important factors that may contribute to PPCs were not collected. For example, the smoking status of patients was not collected, nor were individual comorbidities that may account for some of the differences seen between the high-risk cohorts. The PPOSSUM score does adjust the score dependent on cardiac and respiratory disease, but the classification is broad and dependent on reliable documentation. However, the ASA scores between the groups were not 
different, suggesting that all patients were subjectively risk assessed appropriately for their pre-existing disease.

\section{Abbreviations}

United Kingdom - U.K

Length of stay - LOS

National Emergency Laparotomy Audit - NELA

American Society of Anaesthesia - ASA

Portsmouth- Physiological and Operative Severity Score for the enumeration of Mortality and morbidity PPOSSUM

Post-operative pulmonary complications - PPCs

National Health Service - NHS

Acute respiratory distress syndrome - ARDS

Electronic patient records - EPR

Melbourne Group Score - MGS

Inter-quartile ranges - IQR

Critical Care unit - CCU

Length of Stay - LOS

Acute Lung Injury - ALI

Positive end-expiratory pressure - PEEP

\section{Declarations}

\section{Ethics approval and consent to participate}

The study did not need ethical approval or research registration. This was confirmed by the online National Research Ethics Survey decision tool completed by the research and development departments at the University of Birmingham.

Consent for publication 
Not applicable

\section{Availability of data and materials}

The datasets used and/or analysed during the current study are available from the corresponding author on reasonable request.

\section{Competing Interests}

The authors declare that they have no competing interests.

\section{Funding}

Not applicable

\section{Author's contributions}

SS helped collect the data and helped write the manuscript. JP oversaw the collection of data, analysed and wrote the manuscript. All authors read and approved the final manuscript.

Acknowledgements

Not applicable

Author's Information

Nothing further to add.

\section{References}

1. Barrow E, Anderson ID, Varley S, Pichel AC, Peden CJ, Saunders DI, et al. Current UK practice in emergency laparotomy. Annals of the Royal College of Surgeons of England. 2013;95(8):599-603.

2. Bhangu A, Fitzgerald JE, Fergusson $S$, Khatri $C$, Holmer $H$, Soreide $K$, et al. Determining universal processes related to best outcome in emergency abdominal surgery: a multicentre, international, prospective cohort study. BMJ Open. 2014;4(10):e006239.

3. team Np. First patient report of the National Emergency Laparotomy Audit. London; 2015.

4. Denu ZA, Yasin MO, Melekie TB, A B. Postoperative Pulmonary Complications and Associated Factors among Surgical Patients. J Anesth Clin Res. 2015;6:554.

5. Serejo LG, da Silva-Junior FP, Bastos JP, de Bruin GS, Mota RM, de Bruin PF. Risk factors for pulmonary complications after emergency abdominal surgery. Respir Med. 2007;101(4):808-13.

6. Council MR. Decision Tool: NHS health research authority; [cited 2014 8/8/2014]. Available from: http://www.hra-decisiontools.org.uk/research/. 
7. Agostini P, Naidu B, Cieslik H, Rathinam S, Bishay E, Kalkat MS, et al. Comparison of recognition tools for postoperative pulmonary complications following thoracotomy. Physiotherapy. 2011;97(4):27883.

8. Kelkar KV. Post-operative pulmonary complications after non-cardiothoracic surgery. Indian J Anaesth. 2015;59(9):599-605.

9. Haines KJ, Skinner EH, Berney S, Austin Health PSI. Association of postoperative pulmonary complications with delayed mobilisation following major abdominal surgery: an observational cohort study. Physiotherapy. 2013;99(2):119-25.

10. Scholes RL, Browning L, Sztendur EM, Denehy L. Duration of anaesthesia, type of surgery, respiratory co-morbidity, predicted V02max and smoking predict postoperative pulmonary complications after upper abdominal surgery: an observational study. Aust J Physiother. 2009;55(3):191-8.

11. McAlister FA, Bertsch K, Man J, Bradley J, Jacka M. Incidence of and risk factors for pulmonary complications after nonthoracic surgery. Am J Respir Crit Care Med. 2005;171(5):514-7.

12. Smetana GW, Lawrence VA, Cornell JE, American College of P. Preoperative pulmonary risk stratification for noncardiothoracic surgery: systematic review for the American College of Physicians. Ann Intern Med. 2006;144(8):581-95.

13. Wang T, Derhovanessian A, De Cruz S, Belperio JA, Deng JC, Hoo GS. Subsequent infections in survivors of sepsis: epidemiology and outcomes. J Intensive Care Med. 2014;29(2):87-95.

14. Futier E, Constantin JM, Paugam-Burtz C, Pascal J, Eurin M, Neuschwander A, et al. A trial of intraoperative low-tidal-volume ventilation in abdominal surgery. N Engl J Med. 2013;369(5):428-37.

15. Hemmes SN, Gama de Abreu M, Pelosi P, Schultz MJ. High versus low positive end-expiratory pressure during general anaesthesia for open abdominal surgery (PROVHILO trial): a multicentre randomised controlled trial. Lancet. 2014;384(9942):495-503.

16. Patel JM, Baker R, Yeung J, Small C, West Midlands-Trainee R, Audit N. Intra-operative adherence to lung-protective ventilation: a prospective observational study. Perioper Med (Lond). 2016;5:8-.

17. Futier E, Paugam-Burtz C, Constantin J-M, Pereira B, Jaber S. The OPERA trial - comparison of early nasal high flow oxygen therapy with standard care for prevention of postoperative hypoxemia after abdominal surgery: study protocol for a multicenter randomized controlled trial. Trials. 2013;14:341-.

\section{Tables}


Table 1

Demographics and risk stratification of our cohort are showing in this table. The table demonstrates high risk patients ended up having significantly worse outcomes than low risk patients.

\begin{tabular}{|c|c|c|c|c|}
\hline & $\begin{array}{l}\text { All Patients } \\
\mathrm{N}=362\end{array}$ & $\begin{array}{l}\text { Low Risk } \\
\text { Patients } \\
\mathrm{N}=136\end{array}$ & $\begin{array}{l}\text { High-Risk } \\
\text { Patients } \\
\mathrm{N}=226\end{array}$ & p-values \\
\hline Age & $\begin{array}{l}62(45-74) \\
\text { years }\end{array}$ & $48(38-66)$ & $67(51-77)$ & $<0.001$ \\
\hline Sex, male:female (\%) & $47: 53$ & 49:51 & $44: 58$ & \\
\hline ASA & $37 \%(132)$ & $100 \%$ & $24 \%(54)$ & \\
\hline \multicolumn{4}{|l|}{$\geq 3$} & \\
\hline P-POSSUM (mortality) & $6 \%(2.3-19.3 \%)$ & $2 \%(1.1-3.1 \%)$ & $10.6(5.6-31.3)$ & $<0.001$ \\
\hline ICU admission: & $11 \%(40)$ & 0 & $34(20 \%)$ & \\
\hline $\begin{array}{l}\text { P-POSSUM 5-10\% (N = } \\
72)\end{array}$ & $37 \%(134)$ & 0 & $123(72 \%)$ & \\
\hline $\begin{array}{l}\text { P-POSSUM > 10\% (N = } \\
\text { 156) }\end{array}$ & $48 \%(174)$ & Total (0) & Total (170) & \\
\hline Total (232) & & & & \\
\hline Length of stay & $13(6-25)$ days & $9(6-15)$ days & 17 (9-32) days & $<0.001$ \\
\hline 30-day Mortality & $12 \%(44)$ & $1.4 \%(2)$ & $14 \%(42)$ & $<0.001$ \\
\hline PPC & $30 \%$ & $6 \%$ & $37 \%$ & $<0.001$ \\
\hline
\end{tabular}


Table 2

Table showing the differences in demographics between patients who developed a PPC after laparotomy vs patients who did not.

\begin{tabular}{|llll|}
\hline \multicolumn{4}{|l|}{ Demographics of patients with a PPC and without a PPC } \\
\hline High Risk Laparotomies & No PPC & PPC & p-value \\
& $\mathbf{N}=142$ & $\mathbf{N}=\mathbf{8 4}$ & \\
\hline Age, years & $67(51-77)$ & $68.5(51.5-78)$ & 0.88 \\
\hline P-POSSUM \%(mortality) & $12.3(6.5-31.2)$ & $19.4(7.4-41.6)$ & 0.07 \\
\hline No dyspnoea (n) & $67 \%(92)$ & $62 \%(52)$ & $0.61 *$ \\
\hline No failure (n) & $53 \%(96)$ & $61 \%(51)$ & $0.69 *$ \\
\hline Admission to ICU (n) & $71 \%(101)$ & $85 \%(71)$ & $0.02^{*}$ \\
\hline
\end{tabular}

\section{Figures}

\section{Melbourne Group Score Factors}

- $\mathrm{SpO} 2<90 \%$ on $21 \% \mathrm{FiO}_{2}$

- $W C C>11.2 \times 10^{9} / \mathrm{I}$

- Temperature $>38 \mathrm{C}$

- Purulent sputum differing from pre-operative status (yellow/green)

- Positive signs of infection from sputum microbiology

- Chest radiograph: atelectasis or consolidation

- Physician diagnosed pneumonia

- Prolonged critical care stay/re-admission for respiratory complications

\section{Figure 1}

The Melbourne Group Score. A post-operative pulmonary complication can be diagnosed if 4 or more of these factors are present. 


\section{Cohort Breakdown}

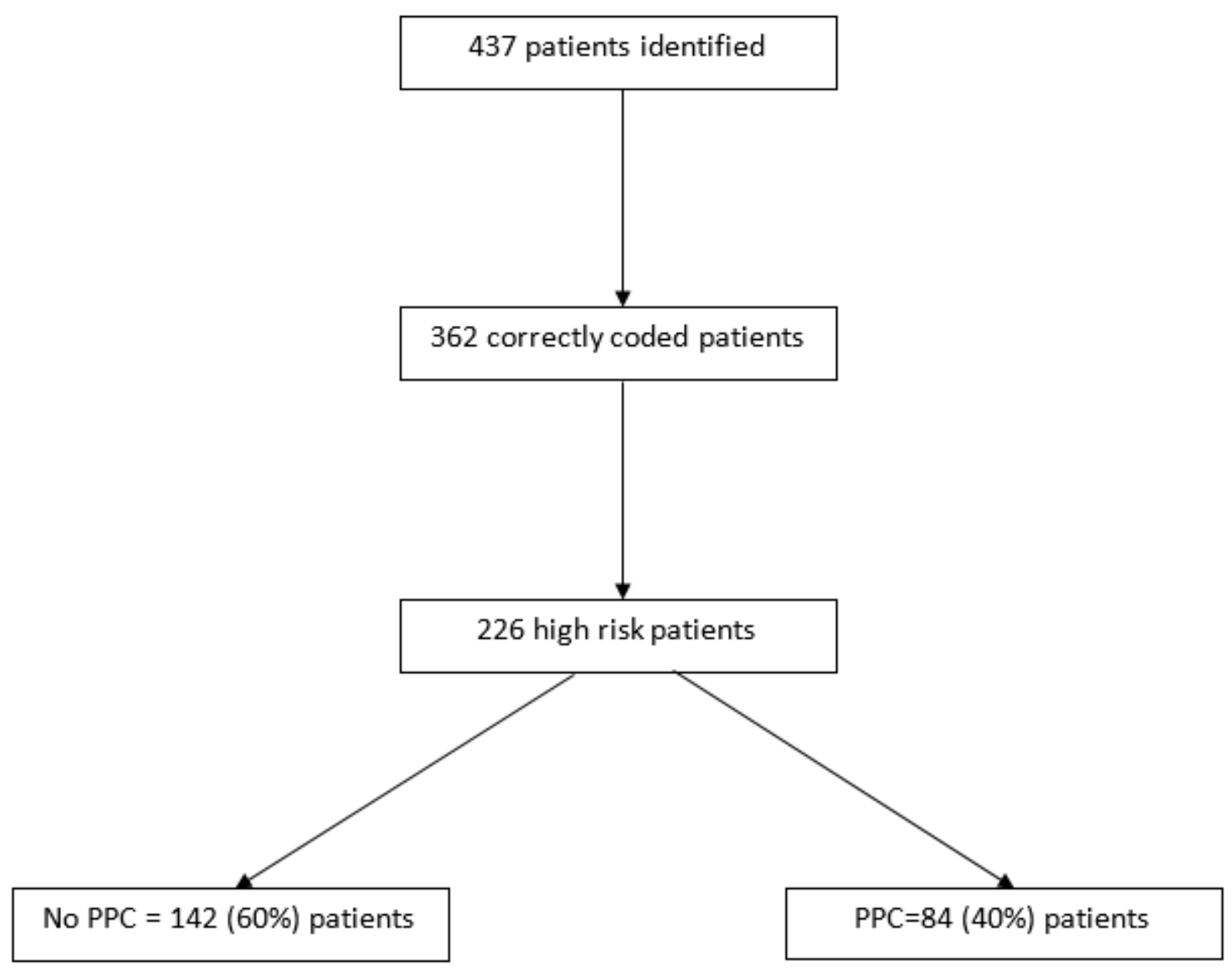

\section{Figure 2}

This image shows an overall summary of our starting cohort being broken down into separate groups. 75 patients were excluded as they were either duplicated or the procedure was incorrectly coded. 


\section{Kaplan Meier Survival Curves}

A

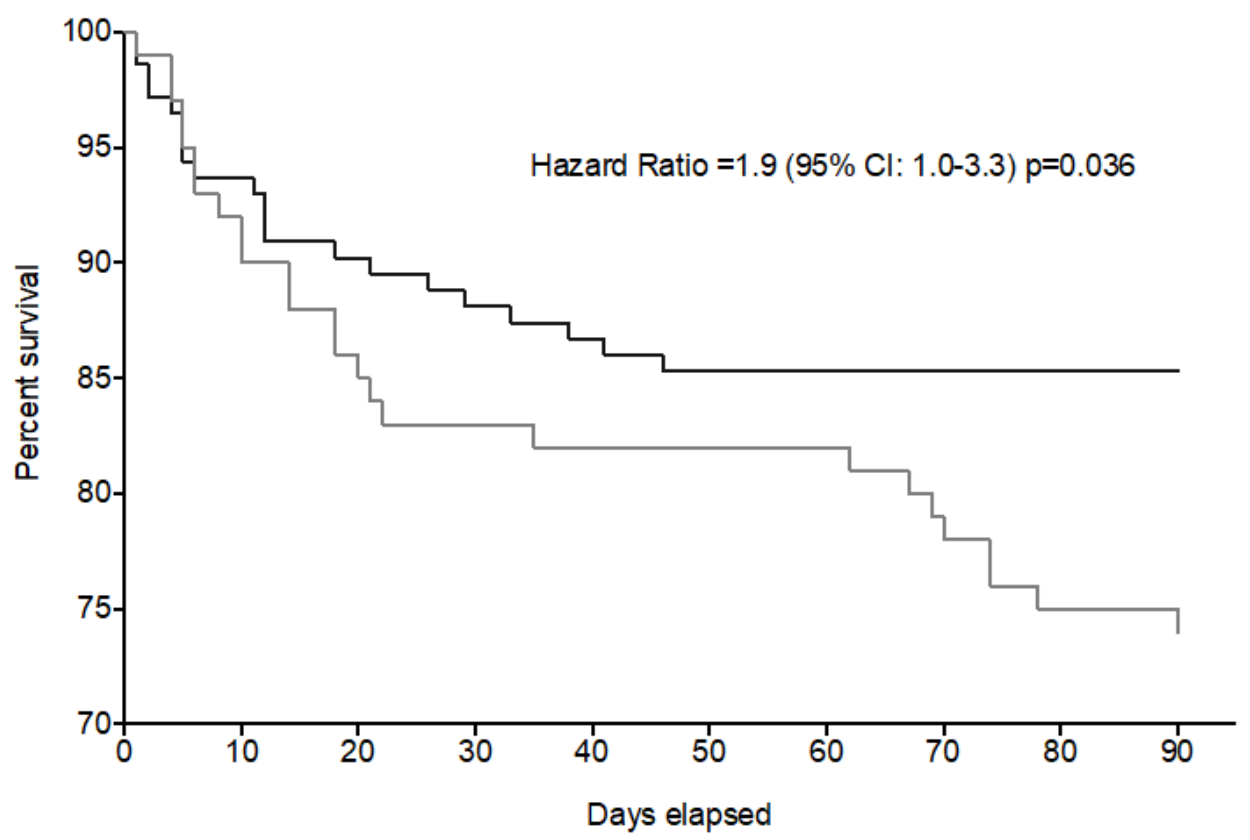

B

$-\mathrm{PPC}$

- NoPPC

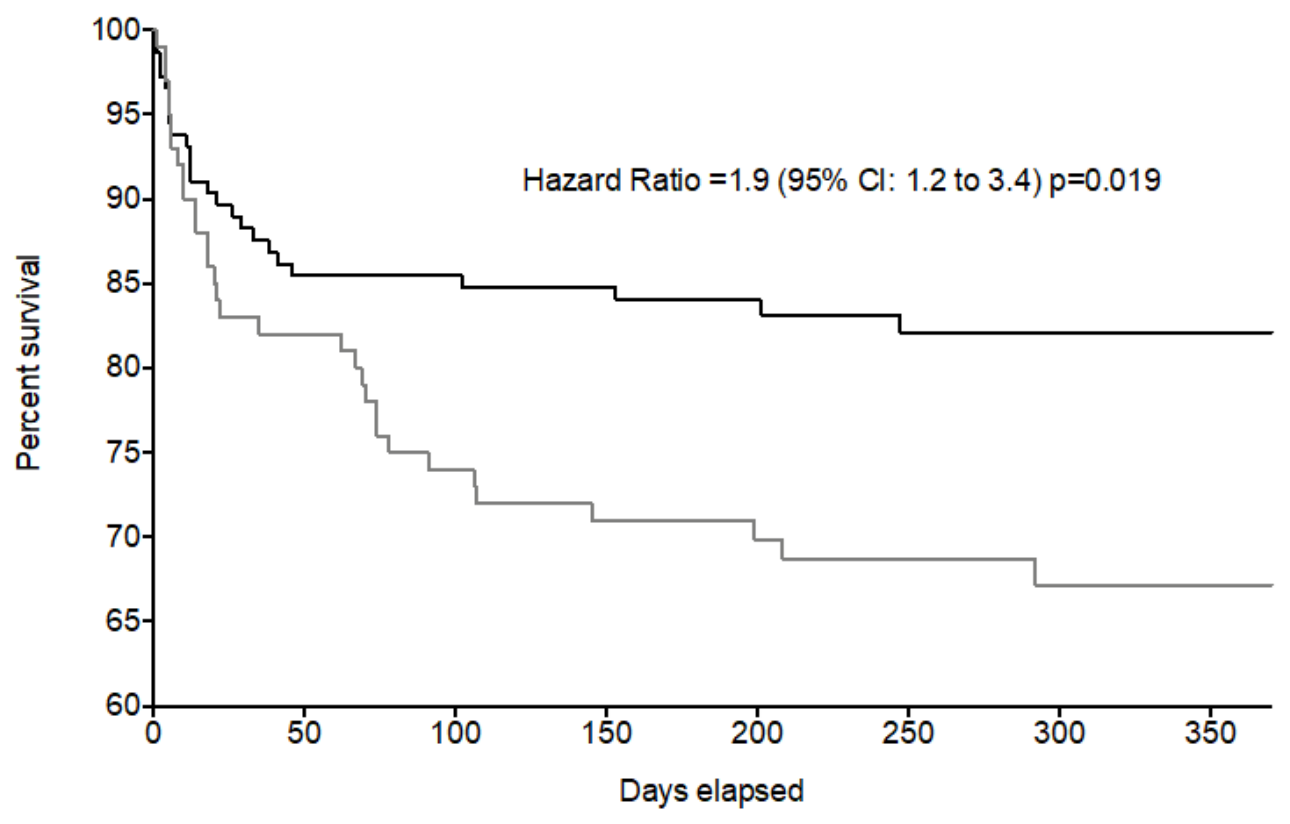

\section{Figure 3}

Kaplan Meier survival curves for our cohort over 360 days. PPC patients had significantly higher 90,180 and 365 day mortality rates.

\section{Supplementary Files}


This is a list of supplementary files associated with this preprint. Click to download.

- PPCSsNELAcopy2.pzfx 